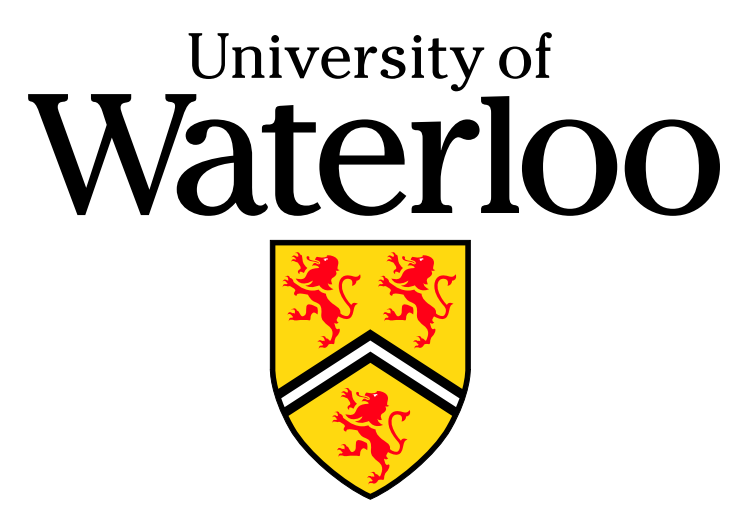

\title{
A New Decentralized Power Allocation Strategy in Single-Hop Wireless Networks
}

Jamshid Abouei, Masoud Ebrahimi, and Amir K. Khandani

Electrical and Computer Engineering Department

University of Waterloo

Waterloo, Ontario, Canada

Technical Report UW-ECE \#2006-26

December, 2006

(C)Jamshid Abouei, Masoud Ebrahimi, and Amir K. Khandani 2006 


\title{
A New Decentralized Power Allocation Strategy in Single-Hop Wireless Networks
}

\author{
Jamshid Abouei, Masoud Ebrahimi, and Amir K. Khandani
}

\begin{abstract}
In this paper, a simple decentralized power allocation strategy is proposed, which relies on the local information in a single-hop wireless network with $n$ links. The main goal of the strategy is to improve the average sum-rate. We first define a new utilitybased framework, in which each user takes into account the negative impact of its power increment on the other users performance. For large $n$ and by knowing only the direct channel gain $h_{i i}$, the optimum strategy for user $i$ is to transmit with full power or remain silent. The transmission policy is to compare $h_{i i}$ with a prespecified threshold $\tau_{n}$ that is a function of $n$. Under a Rayleigh fading channel condition, it is demonstrated that among $n$ pairs of nodes, the average number of active links is of order $\log n$. Also, the average sumrate scales as $\Theta(\log n)$. The performance of the proposed strategy is compared with that of the centralized power allocation scheme and the non-cooperative power control games through simulation and the analytical arguments. The proposed on-off power allocation scheme has the advantage of not requiring a central controller. The proposed strategy relies on a one shot game with a simple structure, rather than the iterative mechanism used in the pricing algorithm. These properties make our scheme more practical in timevarying networks.
\end{abstract}

This work is financially supported by Communications and Information Technology Ontario (CITO), Nortel Networks, and Natural Sciences and Engineering Research Council of Canada (NSERC).

The authors are affiliated with the Coding and Signal Transmission Laboratory, Electrical and Computer Engineering Department, University of Waterloo, Waterloo, ON, N2L 3G1, Canada, Tel: 519-884-8552, Fax: 519-888-4338, Emails: \{jabouei, masoud, khandani\}@cst.uwaterloo.ca. 


\section{Index Terms}

Decentralized power allocation, single-hop wireless network, sum-rate, game theory.

\section{INTRODUCTION}

As the demand for higher data rates increases, effective spectral usage and power control emerge as the main issues in wireless networks. Power control in transmitters has long been regarded as an effective tool to combat channel fluctuations or multipath fading. It also dramatically reduces the interference effect that limits the capacity of the system. In recent years, various power control schemes have been extensively studied in cellular and multihop wireless networks [1]-[12]. For example, in codedivision-multiple-access (CDMA) systems with voice services, the power control is performed to equalize all the received signals at the base station [1]. While, for CDMA data applications or in general multimedia services, the powers are assigned to different users such that the minimum quality of service (QoS) requirements are achieved [2], [3].

In conventional wireless systems, the power allocation is performed by using a central controller. For example, Grandhi et al. [13] propose a centralized power control scheme for the uplink wireless radio systems to achieve a common carrier-tointerference ratio for all the receivers. In the model proposed in [14], a central node jointly controls transmission powers and spreading gains of the users. It is shown that under the bang bang strategy (i.e., the users either transmit with full power or remain silent), the users in the cell are allocated the full powers in decreasing order of the channel gains. Chiang et al. [9] use the geometric programming for the power control in CDMA and multihop wireless networks, and for different values of signal-to-interference-plus-noise ratio (SINR). In the low SINR regime, they solve a nonlinear optimization problem through an iterative algorithm, and based on the cooperation between nodes.

Clearly, centralized power allocation schemes provide a significant improvement 
in the network performance over decentralized approaches. However, they require cooperation between nodes or complete knowledge of all aspects of the network. Also, when the size of the network continues to grow, deploying centralized power allocation schemes becomes computationally intractable. Furthermore, for time-varying networks, due to overwhelming amount of information, the power assignments can not perfectly trace the channel fluctuations. These problems has drawn the attention of the researchers to the decentralized power allocation schemes.

The main goal of applying the decentralized scenario is that the operational decisions are made solely by the individual users. Among many decentralized power control schemes, the problem of the non-cooperative power control game (NPCG) in wireless networks has received much attention in recent years [4]-[8]. The first study on NPCG algorithms in CDMA wireless data networks was framed in [4]. This work was further expanded in [5] and [6] by using the pricing mechanism from game theory [15]. In these works and with the assumption of a static wireless model, they use an iterative algorithm that converges to the optimum powers. Etkin and Tse [7] propose power and spectrum allocation strategies based on game theory concepts. They present non-cooperative power allocation scenarios by using the static and the repeated games. However, they assume omniscient nodes with complete information of the network. In order to analyze the more realistic networks, it is essential to properly account for the unavoidable uncertainty in channel gains and also the interference. This is done by using the Bayesian game theory concept [15], where each user observes the local information in a network and has beliefs about the other users' actions. This motivates us to design a decentralized power allocation strategy for single-hop wireless networks, in which each link utilizes the local information to determine its power.

In this paper, a utility-based framework is proposed that relies on a decentralized power allocation scheme. Unlike [16], in our algorithm, we do not use relay nodes. Also, we consider only one shot game. The main advantage of our scheme is that each user allocates its power based only on its direct channel gain. The above properties 
make our algorithm more practical in time-varying networks. It is shown that for large $n$ and by knowing only the direct channel gain $h_{i i}$, the optimum strategy for user $i$ is to transmit data with full power or remain silent. The decision rule is to compare $h_{i i}$ with a chosen threshold $\tau_{n}$. This approach is different from the multiuser diversity scheme, introduced by Knopp and Humblet [17], where the base station permits only one user with the best channel to transmit. Under a Rayleigh fading channel condition, an asymptotic analysis is carried out to show that among $n$ pairs of nodes, the average number of active links is of $\operatorname{order} \log n$. Also, it is demonstrated that the average sum-rate scales as $\Theta(\log n)$. The performance of the proposed algorithm is compared with that of the centralized power allocation algorithm and NPCG with the pricing scheme through the simulation and the analytical arguments.

The rest of the paper is organized as follows. In Section II, the network model is introduced. The on-off power allocation strategy is presented in Section III. We derive an asymptotic expression for the average sum-rate in Section IV. In Section $\mathrm{V}$, the system performance is characterized and simulated. Finally, in Section VI, an overview of the results and conclusions is presented.

Knuth's notation [18]: For any functions $f(n)$ and $g(n)$ :

- $f(n)=O(g(n))$ means that $\lim _{n \rightarrow \infty}|f(n) / g(n)|<\infty$.

- $f(n)=\omega(g(n))$ means that $\lim _{n \rightarrow \infty}|f(n) / g(n)|=\infty$.

- $f(n)=o(g(n))$ means that $\lim _{n \rightarrow \infty}|f(n) / g(n)|=0$.

- $f(n)=\Theta(g(n))$ means that $\lim _{n \rightarrow \infty}|f(n) / g(n)|=c$, where $0<c<\infty$.

Also, $\mathcal{N}$ is a set of natural numbers less than or equal to $n$.

\section{NeTWORK MODEL}

Consider a single-hop wireless network, in which $n$ pairs of nodes ${ }^{1}$, indexed by $1, \ldots, n$, are located within the network area (Fig. 1). We assume that the number

\footnotetext{
${ }^{1}$ In this work, the term "pair" is used for the transmitter and the related receiver, and "user" only for the transmitter.
} 


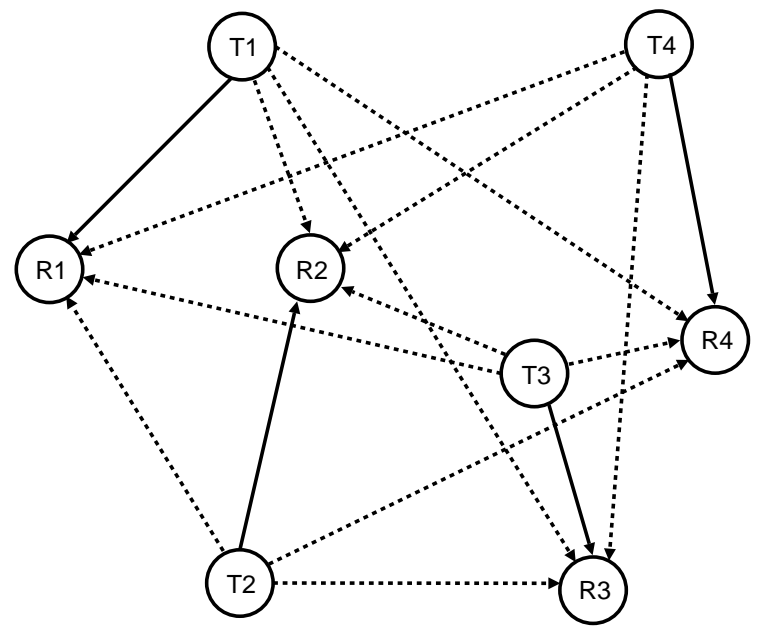

Fig. 1. A single-hop wireless network with $n=4$.

of links is a common information for users in the network. Each pair consists of one transmitter and one receiver equipped with a single antenna. Also, receivers are conventional linear receivers without any multiuser detection and interference cancelation. It is assumed that all the transmissions occur over the same bandwidth. The channel is quasi-static Rayleigh fading. In this model, the channel gains remain constant while transmitting one block and change independently from block to block. The link between transmitter $j$ and receiver $i$ is represented by the channel gain $h_{j i}=\left|g_{j i}\right|^{2}$, where the complex variables $g_{j i}$ 's are the channel coefficients. Under a Rayleigh fading channel condition, $h_{j i}$ 's are exponentially distributed with unit mean. Thus, the cumulative distribution function $(\mathrm{CDF})$ of $h_{j i}$ be denoted by $F_{Y}(y)=1-e^{y}$. Here, we assume that each receiver knows only its direct channel gain. This channelstate information (CSI) is fed back to the corresponding transmitter without any error.

We denote the average transmit power of user $i$ by $p_{i} \in \mathcal{P}$, where $\mathcal{P} \triangleq\left[0, P_{\text {max }}\right]$ is the strategy space of each user. Also, the non-negative vector $\mathbf{P}=\left(p_{1}, \ldots, p_{n}\right)$ represents the vector of all the users' power in the network. The power of additive 
white Gaussian noise (AWGN) in each receiver is assumed to be $N_{0}$. To simplify the notations, we assume $N_{0}$ is normalized by $P_{\max }$. Hence, without loss of generality, in the sequel, we assume $P_{\max }=1$. The interference from other users on receiver $i$ is equal to

$$
I_{i}\left(\mathbf{P}_{-i}\right)=\sum_{\substack{j \neq i \\ j=1}}^{n} h_{j i} p_{j}
$$

where $\mathbf{P}_{-i} \triangleq\left(p_{1}, \ldots, p_{i-1}, p_{i+1}, \ldots, p_{n}\right)$. Throughout the paper, we occasionally use $I_{i}$ instead of $I_{i}\left(\mathbf{P}_{-i}\right)$. Assuming Gaussian signal transmission from all the transmitters, the distribution of the interference will be Gaussian as well. Thus, the SINR of link $i$ is defined as

$$
\gamma_{i}(\mathbf{P}) \triangleq \frac{h_{i i} p_{i}}{I_{i}\left(\mathbf{P}_{-i}\right)+N_{0}} .
$$

The SINR is an important quantity, since it determines a different measure of QoS, particularly the maximum possible data rate. The achievable data rate of link $i$ in the presence of AWGN is obtained as [19]

$$
R_{i}(\mathbf{P})=\log \left(1+\gamma_{i}(\mathbf{P})\right), \quad \text { nats/channel use, }
$$

where $\log ($.$) is the natural logarithm function. As will be seen in the next section,$ the rate function in (3) is used to define our utility function.

\section{Power Allocation Strategy}

In this section, a simple decentralized power allocation strategy based on local information is proposed. We use a utility-based framework inspired by the noncooperative power control game with pricing ${ }^{2}$ proposed in [6]. In our scheme, each user

${ }^{2}$ The NPCG with pricing is an effective strategy to encourage the transmitters to use their powers more efficiently rather than increasing the power selfishly. In the case of the linear pricing, the utility function of link $i$ is defined as

$$
u_{i}(\mathbf{P}) \triangleq R_{i}(\mathbf{P})-\beta_{n} p_{i}, \quad i \in \mathcal{N},
$$

where $\beta_{n}$ is the pricing factor that depends on the number of users. 
aims to maximize its own utility function. Also, the main goal of the proposed strategy is to improve the average sum-rate. To prevent the users from selfishly increasing their powers, the negative impact that each user imposes on the other users should be considered in the definition of the utility function. To this end, the utility function of link $i$ is defined as the rate of link $i$ plus the statistical average rates of the other links in the network. This is different from the pricing scheme in [6], in which the utility of user $i$ is defined as the rate minus a scale factor times the power. Another difference is that we use only one shot game rather than the iterative algorithm used in pricing mechanism.

To obtain the optimum power $p_{i}, i \in \mathcal{N}$, link $i$ relies only on its own channel gain, $h_{i i}$. Here, $h_{i i}$ and $p_{i}$ are the private information of link $i$. However, the private information of other links (their powers and direct channel gains), and also the channel gains $h_{j i}, j \neq i$, are unknown to user $i$. Motivated by the static Bayesian game theory ${ }^{3}$, user $i$ considers the private information of the other links as random variables and selects its power such that its utility function defined in the following is maximized. To this end, the utility function of link $i$ is defined as

$$
u_{i}\left(p_{i}, h_{i i}\right) \triangleq \mathcal{T}\left(p_{i}, h_{i i}\right)+\sum_{\substack{j \neq i \\ j=1}}^{n} \mathcal{S}_{j}\left(p_{i}\right), \quad i \in \mathcal{N}
$$

where

$$
\mathcal{T}\left(p_{i}, h_{i i}\right)=\mathbb{E}\left[\log \left(1+\frac{h_{i i} p_{i}}{I_{i}+N_{0}}\right)\right]
$$

and the expectation is computed with respect to $I_{i}$, and

$$
\mathcal{S}_{j}\left(p_{i}\right)=\mathbb{E}\left[\log \left(1+\frac{h_{j j} p_{j}}{h_{i j} p_{i}+\sum_{k \neq j, i} h_{k j} p_{k}+N_{0}}\right)\right], \quad j \neq i,
$$

${ }^{3}$ In the static Bayesian game theory [15], user $i$ selects the best action $a_{i}$ in the strategy space $\mathcal{A}_{i}$ such that the expected utility function is maximized, i.e.,

$$
a_{i}^{*}\left(\Theta_{i}\right)=\arg \max _{a_{i} \in \mathcal{A}_{i}} \mathbb{E}_{\boldsymbol{\theta}_{-i}}\left[u_{i}\right],
$$

where $\Theta_{i}$ is the private information or type of user $i$ and $\boldsymbol{\theta}_{-i} \triangleq\left(\theta_{1}, \ldots \theta_{i-1}, \theta_{i+1}, \ldots, \theta_{n}\right)$. 
where the expectation is computed with respect to $\mathbf{P}_{-i}$ and $h_{l j}$, for all $l \in \mathcal{N}$. Noting that the power distribution strategy for each user is based on its direct channel gain, we assume all users follow the same power allocation policy $p_{j}=\mathcal{F}\left(h_{j j}\right)$, for all $j \in \mathcal{N}$. We will later describe the function $\mathcal{F}($.$) . Since h_{j j}$ 's have the same distributions, it is concluded that the power $p_{j}$ 's will also have the same distribution.

Noting that the channel gain $h_{k j}$ 's are random variables with the same distributions, $\mathcal{S}_{j}$ becomes independent of $j$. Thus, in the sequel, we drop index $j$ from $\mathcal{S}_{j}$. Consequently, the utility function of link $i$ can be simplified as

$$
u_{i}\left(p_{i}, h_{i i}\right)=\mathcal{T}\left(p_{i}, h_{i i}\right)+(n-1) \mathcal{S}\left(p_{i}\right) .
$$

In the proposed strategy, user $i$ chooses the transmission power $p_{i}$ such that its utility function is maximized, i.e.,

$$
p_{i}^{*}=\arg \max _{p_{i} \in \mathcal{P}} u_{i}\left(p_{i}, h_{i i}\right), \quad i \in \mathcal{N} .
$$

Lemma 1: Let $p_{j}$ be a random variable with mean $q$ and variance $\sigma^{2}$. Then, for the interference $I_{i}=\sum_{j \neq i}^{n} h_{j i} p_{j}$, with probability one (w. p. 1), we have

$$
I_{i} \sim(n-1) q,
$$

as $n \rightarrow \infty$.

Proof: See Appendix I.

Theorem 1: For sufficiently large $n$, if each user knows only its own channel gain, then the optimum power for (8) is one of the extreme points ${ }^{4}$ of $\mathcal{P}$.

Proof: Here, we assume the powers $p_{j}$ are random variables with $\mathbb{E}\left[p_{j}\right]=q$ and variance $\sigma^{2}$. For given $h_{i i}$ and $p_{i}$, Lemma 1 yields

$$
\log \left(1+\frac{h_{i i} p_{i}}{I_{i}+N_{0}}\right) \sim \log \left(1+\frac{h_{i i} p_{i}}{(n-1) q+N_{0}}\right), \quad \text { w. p. } 1,
$$

${ }^{4}$ In the power domain $\mathcal{P}=[0,1]$, the extreme points are 0 and 1. 
as $n \rightarrow \infty$. Thus from $(5), \mathcal{T}\left(p_{i}, h_{i i}\right)$ is simplified as

$$
\begin{aligned}
\mathcal{T}\left(p_{i}, h_{i i}\right) & \approx \mathbb{E}\left[\log \left(1+\frac{h_{i i} p_{i}}{(n-1) q+N_{0}}\right)\right] \\
& =\log \left(1+\frac{h_{i i} p_{i}}{(n-1) q+N_{0}}\right) .
\end{aligned}
$$

With a similar argument, (6) can be simplified as

$$
\mathcal{S}\left(p_{i}\right) \approx \mathbb{E}\left[\log \left(1+\frac{h_{j j} p_{j}}{h_{i j} p_{i}+(n-2) q+N_{0}}\right)\right],
$$

where the expectation is computed with respect to $h_{j j}, h_{i j}$ and $p_{j}$. For sufficiently large $n$,

$$
\begin{aligned}
\frac{h_{i i} p_{i}}{(n-1) q+N_{0}} & \ll 1, \\
\frac{h_{j j} p_{j}}{h_{i j} p_{i}+(n-2) q+N_{0}} & \ll 1 .
\end{aligned}
$$

Consequently, by using $\log (1+z) \approx z$ for $|z| \ll 1$, we have

$$
\begin{gathered}
\mathcal{T}\left(p_{i}, h_{i i}\right) \approx \frac{h_{i i}}{\beta} p_{i}, \\
\mathcal{S}\left(p_{i}\right) \approx \mathbb{E}\left[\frac{h_{j j} p_{j}}{h_{i j} p_{i}+\beta}\right],
\end{gathered}
$$

where $\beta \triangleq n q+N_{0}$. Noting that $h_{j j}$ is independent of $h_{i j}$, and considering $p_{j}=\mathcal{F}\left(h_{j j}\right)$, we have

$$
\begin{aligned}
\mathbb{E}\left[\frac{h_{j j} p_{j}}{h_{i j} p_{i}+\beta}\right] & =\int_{0}^{\infty} \int_{0}^{\infty} \frac{x \mathcal{F}(x)}{y p_{i}+\beta} e^{-x} e^{-y} d x d y \\
& =\int_{0}^{\infty} x \mathcal{F}(x) e^{-x} d x \int_{0}^{\infty} \frac{e^{-y}}{y p_{i}+\beta} d y \\
& =-\frac{\mu}{p_{i}} e^{\frac{\beta}{p_{i}}} \operatorname{Ei}\left(-\frac{\beta}{p_{i}}\right),
\end{aligned}
$$

where $\mu=\int_{0}^{\infty} x \mathcal{F}(x) e^{-x} d x$ is a constant value, and $\operatorname{Ei}(x) \triangleq-\int_{-x}^{\infty} \frac{e^{-t}}{t} d t, x<0$ is the exponential-integral function. An asymptotic expansion of $\operatorname{Ei}(x)$ is as [20]

$$
\operatorname{Ei}(x)=\frac{e^{x}}{x}\left[\sum_{k=0}^{M-1} \frac{k !}{x^{k}}+O\left(|x|^{-M}\right)\right] ; \quad M=1,2, \ldots,
$$


as $x \rightarrow-\infty$. By using (13), (14) and setting $M=3$, we can rewrite (12) as

$$
\mathcal{S}\left(p_{i}\right) \approx \frac{\mu}{\beta}\left(1-\frac{p_{i}}{\beta}+2\left(\frac{p_{i}}{\beta}\right)^{2}\right)+\frac{\mu}{\beta} O\left(\left|\frac{p_{i}}{\beta}\right|^{3}\right)
$$

For large values of $n$, we can ignore the term $O\left(\left|\frac{p_{i}}{\beta}\right|^{3}\right)$. Consequently, an asymptotic formula for the proposed utility function (7) is,

$$
\begin{aligned}
u_{i}\left(p_{i}, h_{i i}\right) & =\mathcal{T}\left(p_{i}, h_{i i}\right)+(n-1) \mathcal{S}\left(p_{i}\right) \\
& \approx \frac{h_{i i}}{\beta} p_{i}+\frac{n \mu}{\beta}\left(1-\frac{p_{i}}{\beta}+2\left(\frac{p_{i}}{\beta}\right)^{2}\right) .
\end{aligned}
$$

Taking the first-order derivative of the utility in (16) yields,

$$
\frac{\partial u_{i}\left(p_{i}, h_{i i}\right)}{\partial p_{i}}=\frac{1}{\beta}\left(h_{i i}-\frac{n \mu}{\beta}\right)+\left(\frac{2}{\beta}\right)^{2} \frac{n \mu}{\beta} p_{i} .
$$

Also, the second-order derivative of the utility in (16), $\frac{\partial^{2} u_{i}\left(p_{i}, h_{i i}\right)}{\partial p_{i}^{2}}=\left(\frac{2}{\beta}\right)^{2} \frac{n \mu}{\beta}$, is a positive value. Thus, (16) is a convex function of $p_{i}$. It is a known fact that a convex function attains its maximum at one of the extreme points of its domain [21]. This completes the proof of the theorem.

Corollary 1: For the proposed algorithm and sufficiently large $n$, the power distribution for each user is a Bernoulli random variable with parameter $q$, i.e.,

$$
f\left(p_{i}\right)= \begin{cases}q, & p_{i}=1 \\ 1-q, & p_{i}=0\end{cases}
$$

for all $i \in \mathcal{N}$

Motivated by Corollary 1 and with the assumption that each user knows its direct channel gain, we describe the on-off power allocation strategy for single-hop wireless networks.

On-Off Power Allocation Strategy: In the network with $n$ links, all users perform the following steps during one block: 
1- Based on the direct channel gain, the transmission policy for each user is

$$
p_{i}=\mathcal{F}\left(h_{i i}\right)= \begin{cases}1, & \text { if } h_{i i}>\tau_{n} \\ 0, & \text { Otherwise }\end{cases}
$$

for all $i \in \mathcal{N}$, where $\tau_{n}$ is a prespecified threshold level.

2- After adjusting the powers, each active user transmits a pilot signal with full power. The receivers measure the interference and compute the SINR and the rate using (3). Then, each receiver feedbacks the rate to its transmitter.

3- The active user transmits data with the computed rate and with full power.

\section{Network Analysis with On-Off Power Strategy}

As described in the previous section, in the on-off power allocation strategy, each user transmits with full power if its direct channel gain is greater than $\tau_{n}$. Noting that each link is activated independent of the other links with probability $q$, it is concluded that the power distribution for each user is a Bernoulli random variable with parameter $q$. Also, the number of active links is a binomial random variable with parameters $n$ and $q$. Here, we denote $q$ as the probability of the link activation that is defined as

$$
q \triangleq \operatorname{Pr}\left\{h_{i i}>\tau_{n}\right\}
$$

In this section, we use the average sum-rate as a key parameter in the network performance. To justify using the average, it should be noted that under the quasistatic channel model, the channel gains and the interference change independently from block to block. So, it should be considered the average sum-rate instead of the sum-rate, where the average is computed with respect to $h_{i i}$ 's and $I_{i}$ 's. Thus, letting $\bar{R}_{\text {sum }}$ denote the average sum-rate of the network, we have

$$
\bar{R}_{\text {sum }}=\mathbb{E}\left[\sum_{i=1}^{n} R_{i}\right]=\sum_{i=1}^{n} \mathbb{E}\left[R_{i}\right]
$$

In the next theorem, we derive asymptotically the optimum threshold $\tau_{n}$ such that the average sum-rate is maximized. 
Theorem 2: For the on-off power scheme and sufficiently large $n$, the optimum $\tau_{n}$ that maximizes the average sum-rate is

$$
\tau_{n}^{*}=\log n-\log \log n+\Theta(1) .
$$

Proof: For the on-off power scheme and using (19) and (20), we have

$$
\begin{aligned}
\mathbb{E}\left[R_{i}\right] & =\mathbb{E}\left[R_{i} \mid h_{i i}>\tau_{n}\right] \operatorname{Pr}\left\{h_{i i}>\tau_{n}\right\}+\mathbb{E}\left[R_{i} \mid h_{i i} \leq \tau_{n}\right] \operatorname{Pr}\left\{h_{i i} \leq \tau_{n}\right\} \\
& =q \mathbb{E}\left[R_{i} \mid h_{i i}>\tau_{n}\right]+(1-q) \mathbb{E}\left[R_{i} \mid h_{i i} \leq \tau_{n}\right] .
\end{aligned}
$$

Noting that for $h_{i i} \leq \tau_{n}, p_{i}=0$, it is concluded

$$
\mathbb{E}\left[R_{i} \mid h_{i i} \leq \tau_{n}\right]=\mathbb{E}\left[\log \left(1+\frac{h_{i i} p_{i}}{I_{i}+N_{0}}\right) \mid h_{i i} \leq \tau_{n}\right]=0 .
$$

Thus,

$$
\mathbb{E}\left[R_{i}\right]=q \mathbb{E}\left[\log \left(1+\frac{h_{i i}}{I_{i}+N_{0}}\right) \mid h_{i i}>\tau_{n}\right]
$$

By using Lemma 1, we have

$$
\mathbb{E}\left[R_{i}\right] \approx q \mathbb{E}\left[\log \left(1+\frac{h_{i i}}{(n-1) q+N_{0}}\right) \mid h_{i i}>\tau_{n}\right],
$$

where the expectation is computed with respect to $h_{i i}$. For large values of $n$ and by using $\log (1+z) \approx z$ for $|z| \ll 1$, it yields

$$
\begin{aligned}
\mathbb{E}\left[R_{i}\right] & \approx q \mathbb{E}\left[\frac{h_{i i}}{n q+N_{0}} \mid h_{i i}>\tau_{n}\right] \\
& =\frac{q}{n q+N_{0}} \int_{\tau_{n}}^{\infty} x e^{-\left(x-\tau_{n}\right)} d x \\
& =\frac{q\left(1+\tau_{n}\right)}{n q+N_{0}} .
\end{aligned}
$$

Consequently, by using $q=\operatorname{Pr}\left\{h_{i i}>\tau_{n}\right\}=e^{-\tau_{n}}$, (20) is expressed as

$$
\begin{aligned}
\bar{R}_{\text {sum }} & \approx \sum_{i=1}^{n} \frac{q\left(1+\tau_{n}\right)}{n q+N_{0}} \\
& =n \frac{e^{-\tau_{n}}\left(1+\tau_{n}\right)}{n e^{-\tau_{n}}+N_{0}} .
\end{aligned}
$$

Thus, the optimization problem is

$$
\tau_{n}^{*}=\arg \max _{\tau_{n}} \bar{R}_{\text {sum }}
$$


Taking the first-order derivative of (22) yields

$$
\frac{\partial \bar{R}_{\text {sum }}}{\partial \tau_{n}}=n \frac{n e^{-2 \tau_{n}}-N_{0} \tau_{n} e^{-\tau_{n}}}{\left(n e^{-\tau_{n}}+N_{0}\right)^{2}} .
$$

Also, the second-order derivative of (22),

$$
\frac{\partial^{2} \bar{R}_{\text {sum }}}{\partial \tau_{n}^{2}} \approx-n^{2} \frac{N_{0}\left(2+\tau_{n}\right) e^{-2 \tau_{n}}}{\left(n e^{-\tau_{n}}+N_{0}\right)^{3}},
$$

is a negative value. Thus, the maximum value of $\bar{R}_{\text {sum }}$ is obtained by setting (24) equal to zero. So, we have

$$
n e^{-\tau_{n}}-N_{0} \tau_{n}=0
$$

or

$$
n q+N_{0} \log q=0
$$

It can be verified that the solution for $(25)$ is

$$
q^{*}=N_{0} \frac{\log n}{n}\left(1-\frac{\log \log n}{\log n}-\frac{\log N_{0}}{\log n}\right) .
$$

Consequently, the optimum threshold level $\tau_{n}$ that maximizes $\bar{R}_{\text {sum }}$ is

$$
\begin{aligned}
\tau_{n}^{*} & =-\log q^{*} \\
& =\log n-\log \log n+\Theta(1) .
\end{aligned}
$$

Corollary 2: For the proposed strategy, the probability of the link activation scales as $\Theta\left(\frac{\log n}{n}\right)$.

Corollary 3: In the network with $n$ pairs of nodes, the average number of active links is of order $\log n$.

Theorem 3: For the on-off power allocation strategy, the average sum-rate scales as $\Theta(\log n)$.

Proof: By using (22), the average sum-rate for every value of $\tau_{n}$ is

$$
\begin{aligned}
\bar{R}_{\text {sum }} & \approx n \frac{e^{-\tau_{n}}\left(1+\tau_{n}\right)}{n e^{-\tau_{n}}+N_{0}} \\
& =n \frac{q(1-\log q)}{n q+N_{0}} .
\end{aligned}
$$


Fot sufficiently large $n, n q+N_{0} \approx n q$. Thus, (29) can be simplified as

$$
\bar{R}_{\text {sum }} \approx 1-\log q .
$$

Substituting (26) in (30) yields

$$
\bar{R}_{\text {sum }} \approx \log n-\log \log n+\Theta(1) .
$$

From (28), it is concluded that the average sum-rate is a function of the threshold level $\tau_{n}$, where $\tau_{n}$ depends on the channel model. Also, Theorem 3 implies that for the network with the on-off power allocation scheme, the average sum-rate scales as $\Theta(\log n)$ without using any relay and coordination between nodes.

\section{Performance Evaluation}

In this section, we compare the performance of the proposed scheme with that of the NPCG, the centralized power allocation and NPCG with linear pricing algorithms. In the NPCG, each user aims to maximize its utility function selfishly by adjusting its power. Given the fixed power vector $\mathbf{P}_{-i}$, the utility function $R_{i}(\mathbf{P})=\log \left(1+\gamma_{i}(\mathbf{P})\right)$ is a concave function of power $p_{i}$. Using the Debreu Theorem [22], it can be shown that the NPCG algorithm has a unique Nash Equilibrium (NE) [23] that is the maximum power for each user. Since each transmitter uses full power, the average sum-rate is degraded. The next lemma investigates asymptotically the average sum-rate of the NPCG.

Lemma 2: The average sum-rate of the NPCG scheme is a decreasing function of the number of links, and approaches 1 as $n \rightarrow \infty$.

Proof: See Appendix II.

In the centralized power allocation scheme, it is assumed that the central knows all the network information. For each channel realization and through exhaustive search, the central node selects the optimum power vector $\mathbf{P}=\left(p_{1}, \ldots, p_{n}\right)$ such that the maximum average sum-rate is achieved. 
We also compare the performance of the proposed strategy with that of the NPCG with linear pricing proposed in [6]. Referring to [6], the NPCG with linear pricing is defined as

$$
u_{i}(\mathbf{P}) \triangleq r_{i}(\mathbf{P})-\beta_{n} p_{i}
$$

where $r_{i}(\mathbf{P})$ is the data transmission rate. It is shown that if $u_{i}(\mathbf{P})$ satisfies the NonDecreasing Differences ${ }^{5}$ (NDD) property, there always exists a NE [6]. It is easy to verify that the NDD property is not valid for the utility function (32) with

$$
r_{i}(\mathbf{P})=\log \left(1+\frac{h_{i i} p_{i}}{I_{i}\left(\mathbf{P}_{-i}\right)+N_{0}}\right) .
$$

One solution for the problem is to modify the utility function by dropping 1 in (33), i.e.,

$$
u_{i}(\mathbf{P})=\log \left(\frac{h_{i i} p_{i}}{I_{i}\left(\mathbf{P}_{-i}\right)+N_{0}}\right)-\beta_{n} p_{i}
$$

In this case, user $i$ selects the optimum power $p_{i}$ such that (34) is maximized. We use the optimum powers computed in this manner in the rate (33) to obtain the average sum-rate.

Fig. 2 illustrates the average sum-rates of the on-off power allocation strategy, the NPCG, the centralized power allocation algorithm and the NPCG with linear pricing defined in (34). It is seen that the performance of the proposed strategy is better than that of the NPCG. Also, the highest average sum-rate is achieved by the centralized scheme. However, in the network with a large number of links, deploying centralized power allocation schemes becomes computationally intractable. While in our strategy, the average sum-rate is achieved without coordination among the links.

Also, it is seen that the result for the new developed strategy is better than that of the pricing scheme defined in (34). It should be noted that the proposed strategy uses a one shot game with a simple structure. While in the NPCG with

\footnotetext{
${ }^{5}$ According to supermodularity theory [24], for continuous and twice differentiable utilities, $u_{i}(\mathbf{P})$ has NDD in $\mathbf{P}$ if and only if $\partial^{2} u_{i} / \partial p_{i} \partial p_{j} \geq 0$ for all $j \neq i$. One condition for existence of the Nash equilibria is that the utility function $u_{i}(\mathbf{P})$ has (NDD) in $\mathbf{P}[6]$.
} 


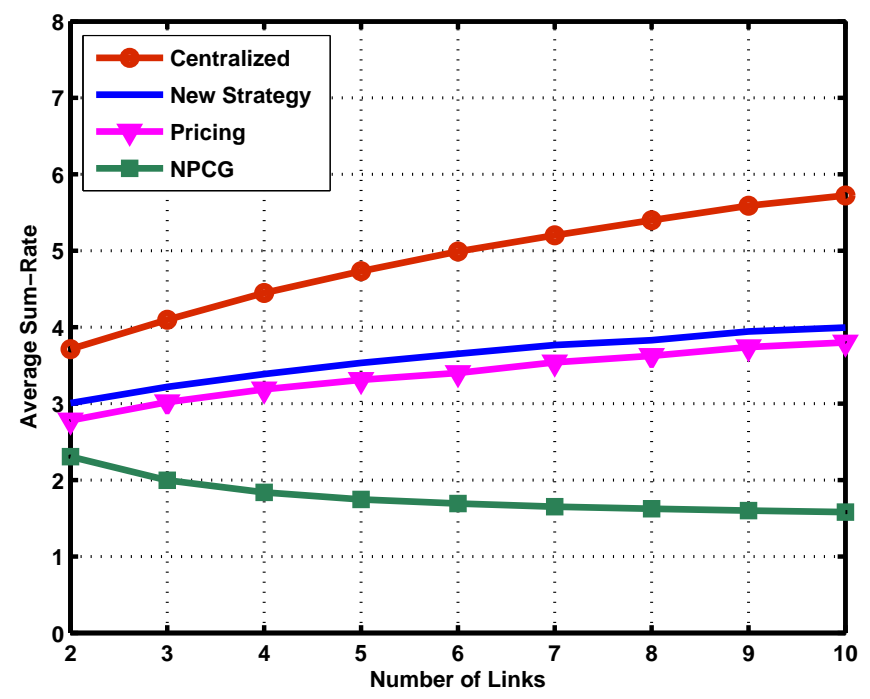

Fig. 2. Average sum-rate vs. the number of links for $N_{0}=0.1$.

pricing, the optimum powers and the pricing factors are obtained by using iterative algorithms. Updating the pricing factor is the other problem in the pricing scheme. Particularly in the networks with mobile nodes, where the channel gains change from block to block, updating the pricing factor in each channel realization is not feasible. The aforementioned properties make our algorithm more practical in time-varying networks.

Fig. 3 shows the average sum-rate of the proposed strategy, the NPCG and the centralized power allocation schemes versus the signal-to-noise ratio (SNR), where

$$
S N R_{i} \triangleq \frac{h_{i i} p_{i}}{N_{0}}
$$

It is seen that the average sum-rate of the proposed strategy increases logarithmically with the SNR.

\section{CONCLUSION}

In this paper, we developed a new decentralized power allocation strategy for single-hop wireless networks. We presented the on-off power allocation strategy, in 


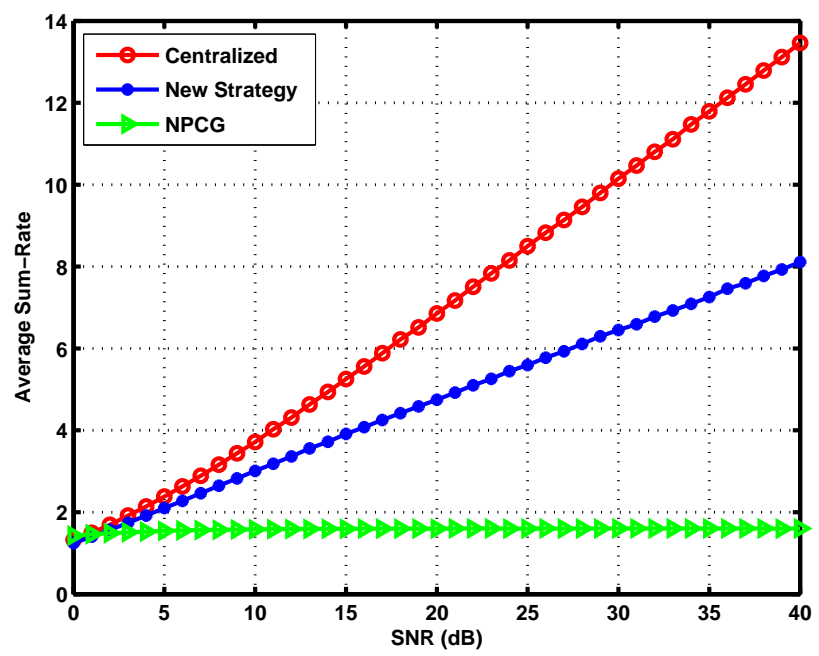

Fig. 3. Average sum-rate vs. SNR for $n=2$.

which each user transmits with full power if its direct channel gain is greater than a threshold level $\tau_{n}$. This policy motivates users remain silent in the case of bad channels in order to adopt a social behavior. It was demonstrated that among $n$ pairs of nodes, the average number of links that communicate simultaneously is of order $\log n$. Also, we proved that the average sum-rate scales as $\Theta(\log n)$ without using relay nodes. It should be noted that decreasing in the average rate per link with $n$ may be regarded as unacceptable by users when the number of links is large. A feasible scenario for solving this problem is to use relay nodes or a partial cooperation between links.

\section{Appendix I}

\section{Proof of Lemma 1}

Let define $\xi_{j}=h_{j i} p_{j}$, where $h_{j i}$ is independent of $p_{j}$. Under a quasi-static Rayleigh fading channel condition, it is concluded that $\xi_{j}$ 's are the i.i.d. random 
variables with

$$
\begin{aligned}
\mathbb{E}\left[\xi_{j}\right] & =\mathbb{E}\left[h_{j i} p_{j}\right]=q, \\
\operatorname{Var}\left[\xi_{j}\right] & =\mathbb{E}\left[\xi_{j}^{2}\right]-\mathbb{E}^{2}\left[\xi_{j}\right]=2 \sigma^{2}+q^{2},
\end{aligned}
$$

where $\mathbb{E}\left[h_{j i}^{2}\right]=2$. Also, the received interference $I_{i}=\sum_{j \neq i} h_{j i} p_{j}$ is a random variable with mean $\mu$ and variance $\vartheta^{2}$, where

$$
\begin{aligned}
\mu & =\mathbb{E}\left[\sum_{j \neq i}^{n} \xi_{j}\right]=(n-1) q, \\
\vartheta^{2} & =\operatorname{Var}\left[\sum_{j \neq i}^{n} \xi_{j}\right]=(n-1)\left(2 \sigma^{2}+q^{2}\right) .
\end{aligned}
$$

By using the Chebyshev inequality [25], we obtain

$$
\operatorname{Pr}\left\{\left|I_{i}-\mu\right|<\psi_{n}\right\} \geq 1-\frac{\vartheta^{2}}{\psi_{n}^{2}},
$$

for all $\psi_{n}>0$. Thus,

$$
\operatorname{Pr}\left\{\left|I_{i}-(n-1) q\right|<\psi_{n}\right\} \geq 1-\frac{(n-1)\left(2 \sigma^{2}+q^{2}\right)}{\psi_{n}^{2}} .
$$

It is seen that for all $\psi_{n}=\omega\left(\sqrt{(n-1)\left(2 \sigma^{2}+q^{2}\right)}\right)$,

$$
\lim _{n \rightarrow \infty} 1-\frac{(n-1)\left(2 \sigma^{2}+q^{2}\right)}{\psi_{n}^{2}}=1 \text {. }
$$

Thus,

$$
(n-1) q-\psi_{n}<I_{i}<(n-1) q+\psi_{n}, \quad \text { w.p. } 1 .
$$

If we choose $\psi_{n}=o((n-1) q)$, then we can assume $I_{i} \sim(n-1) q$, w. p. 1 .

\section{Appendix II}

\section{Proof of Lemma 2}

Noting that all the users transmit data with power $P_{\max }=1$, the average sumrate is

$$
\bar{R}_{\text {sum }}=\mathbb{E}\left[\sum_{i=1}^{n} R_{i}\right]=\sum_{i=1}^{n} \mathbb{E}\left[\log \left(1+\frac{h_{i i}}{I_{i}+N_{0}}\right)\right]
$$


where $I_{i}=\sum_{k \neq i}^{n} h_{k i}$ is the received interference. It is obvious that the average sumrate decreases when the number of users increases. By using Lemma 1 and noting that $\mu=\mathbb{E}\left[I_{i}\right]=n-1$, the average sum-rate is simplified as

$$
\bar{R}_{\text {sum }} \approx \sum_{i=1}^{n} \mathbb{E}\left[\log \left(1+\frac{h_{i i}}{(n-1)+N_{0}}\right)\right],
$$

where the expectation is computed with respect to $h_{i i}$. Consequently, for sufficiently large $n$ and using $\log (1+z) \approx z$ for $|z| \ll 1$, the average sum-rate is

$$
\bar{R}_{\text {sum }} \approx \frac{n}{n+N_{0}} \mathbb{E}\left[h_{i i}\right] \approx 1
$$

and this proves the lemma.

\section{REFERENCES}

[1] K. S. Gilhousen, I. M. Jacobs, R. Padovani, A. J. Viterbi, L. A. Weaver, and C. E. Wheatley III, "On the capacity of a cellular CDMA system," IEEE Trans. on Vehicular Technology, vol. 40, no. 2, pp. 303-312, May 1991.

[2] A. Sampath, P. Sarath Kumar, and J. M. Holtzman, "Power control and resource management for a multimedia CDMA wireless system," in Proc. IEEE PIMRC'95, Sept. 1995, vol. 1, pp. 21-25.

[3] O. Seong-Jun, T. L. Olsen, and K. M. Wasserman, "Distributed power control and spreading gain allocation in CDMA data networks," in Proc. INFOCOM 2000, March 2000, vol. 2, pp. 379-385.

[4] V. Shah, N. B. Mandayam, and D. J. Goodman, "Power control for wireless data based on utility and pricing," in Proc. IEEE PIMRC'98, Sept. 1998, vol. 3, pp. 1427-1432.

[5] D. J. Goodman and N. B. Mandayam, "Power control for wireless data," in IEEE Personal Commun., Feb. 2000, vol. 7, pp. 48-54.

[6] C. U. Saraydar, N. B. Mandayam, and D. J. Goodman, "Efficient power control via pricing in wireless data networks," IEEE Trans. on Commun., vol. 50, no. 2, pp. 291-303, Feb. 2002.

[7] R. Etkin, A. Parekh, and D. Tse, "Spectrum sharing in unlicensed bands," in IEEE Dyspan, Baltimore MD, 2005.

[8] F. Meshkati, M. Chiang, H. V. Poor, and S. C. Schwartz, "A game-theoretic approach to energy-efficient power control in multicarrier CDMA systems," IEEE Journal on Selected Areas in Communications, vol. 24, no. 6, pp. 1115 - 1129, June 2006.

[9] M. Chiang, C. W. Tan, D. Palomar, D. O'Neill, and D. Julian, "Power control by geometric programming," To appear in IEEE Trans. on Wireless Commun., 2006. 
[10] A. Gjendemsj, D. Gesbert, G. E. фien, and S. G. Kiani, "Optimal power allocation and scheduling for two-cell capacity maximization," in Proc. IEEE Symposium on Modeling and Optimization in Mobile, Ad-Hoc and Wireless Networks, Boston, MA, April 2006.

[11] M. Xiao, N. B. Shroff, and E. K. P. Chong, "A utility-based power-control scheme in wireless cellular systems," IEEE/ACM Trans. on Networking, vol. 11, no. 2, pp. 210 - 221, April 2003.

[12] J. Huang, R. A. Berry, and M. L. Honig, "Distributed interference compensation for wireless networks," IEEE Journal on Selected Areas in Communications, vol. 24, no. 5, pp. 1074 - 1084, May 2006.

[13] S. A. Grandhi, R. Vijayan, D. J. Goodman, and J. Zander, "Centralized power control in cellular radio systems," IEEE Trans. on Vehicular Technology, vol. 42, no. 4, pp. 466-468, Nov. 1993.

[14] O. Seong-Jun, D. Zhang, and K. M. Wasserman, "Optimal resource allocation in multiservice CDMA networks," IEEE Trans. on Wireless Commun., vol. 2, no. 4, pp. 811-821, July 2003.

[15] M. J. Osborne, An Introduction to Game Theory, Oxford University Press, 2004.

[16] R. Gowaikar, B. Hochwald, and B. Hassibi, "Communication over a wireless network with random connections," IEEE Trans. on Information Theory, vol. 52, no. 7, pp. 2857-2871, July 2006.

[17] R. Knopp and P. A. Humblet, "Information capacity and power control in single-cell multiuser communications," in Proc. IEEE ICC. Seattle, WA, 1995, pp. 331-335.

[18] D. E. Knuth, "Big omicron and big omega and big theta," in ACM SIGACT News, April-June 1967, vol. 8 , pp. $18-24$.

[19] T. M. Cover and J. A. Thomas, Elements of Information Theory, John Wiley and Sons Inc., 2nd edition, 2006.

[20] I. S. Gradshteyn, I. M. Ryzhik, and A. Jeffrey, Table of Integrals, Series, and Products, Academic Press, 1994.

[21] D. P. Bertsekas, Nonlinear Programming, Athena Scientific, 2nd edition, 1999.

[22] G. Debreu, "A social equilibrium existence theorem," in Proc. of the National Academy of Science, 1952, pp. 886-893.

[23] J. F. Nash, "Non-cooperative games," Ann. Math., vol. 54, pp. 289-295, 1951.

[24] D. M. Topkis, Supermodularity and complementarity, Princeton University Press, Princeton, New Jersey, 1998.

[25] S. M. Ross, Introduction to Probability Models, Academic Press, 8th edition, 2003. 